\title{
GeTe-filled Carbon Nanotubes for Data Storage Applications
}

\author{
Cristina E. Giusca ${ }^{1}$, Vlad Stolojan ${ }^{1}$, Jeremy Sloan ${ }^{2}$, Hidetsugu Shiozawa ${ }^{1}$ and S. Ravi P. Silva ${ }^{1}$ \\ ${ }^{1}$ Nanoelectronics Centre, Advanced Technology Institute, University of Surrey, Guildford, GU2 \\ 7XH, United Kingdom \\ ${ }^{2}$ Department of Physics, University of Warwick, Coventry CV4 7AL, United Kingdom
}

\begin{abstract}
By virtue of their unique electronic properties, nanometer-diameter sized single-walled carbon nanotubes represent ideal candidates to function as active parts of nanoelectronic memory storage devices. We show for the first time that GeTe, a phase change material, currently considered to be one of the most promising materials for data-storage applications, can efficiently be encapsulated within single-walled carbon nanontubes of $1.4 \mathrm{~nm}$ diameter. Structural investigations on the encapsulated GeTe nanowires have been carried out by high resolution transmission electron microscopy. The electronic interactions between the filling material and the host nanotube have been examined using ultraviolet photoelectron spectroscopy experiments and show that the electronic structure of the encapsulating nanotube and that of the encased filling are not perturbed by the presence of each of the other component.

The newly formed hybrids offer potential to operate as active elements in non-volatile electronic memory storage devices.
\end{abstract}

\section{INTRODUCTION}

The past several decades have witnessed a constant growth in the data storage capabilities of silicon-based electronics but it is becoming increasingly tougher to keep up with the industry's long-term trends towards miniaturisation because of fundamental physical limitations preventing current designs from functioning reliably at the nanometer scale. Molecular electronics has the potential to overcome these limitations by providing new designs for building memory devices from the bottom-up using individual molecules.

By virtue of their unique electronic properties, as well as chemical inertness and mechanical toughness, nanometer-diameter sized single-walled carbon nanotubes (SWNTs) represent ideal candidates to function as active components of nanoelectronic memory storage devices. Indeed, designs of a carbon nanotube-based electromechanical universal memory [1], as well as a non-volatile random access memory (RAM) using carbon nanotubes (CNTs) for molecular computing have been proposed [2], showing that these devices present competitive capabilities to existing memory technologies.

In addition to the remarkable physical and chemical properties, due to unique dimensions and geometry, the central cavity of SWNTs has been shown to serve as a template for the controlled growth of one dimensional crystals, providing an unparalleled opportunity of engineering structures with sometimes entirely novel co-ordinations and stereochemistry [3]. A wide range of organic, as well as inorganic materials have been shown to fill the inner cores of SWNTs [4-6], however no reports of phase change materials encapsulated within SWNTs exist. Numerous phase changing materials have been developed, a few examples include GeTe [7], 
SbTe [8], $\mathrm{Ge}_{1} \mathrm{Se}_{1} \mathrm{Te}_{2}$ [9], GeInSbTe and compositional variants of AgInSbTe [10], etc, and their properties investigated to assess their suitability as memory switching components $[11,12]$.

The dimensionality of these systems ranges from thin films [9] to nanowires [13] and to nanoparticles [14]. Diameters of phase change nanowires previously synthesized range between $200 \mathrm{~nm}$ to $20 \mathrm{~nm}$ and depend on the size of the catalyst particle. Therefore, using SWNTs with diameters as low as $1 \mathrm{~nm}$ as templates for creating thinner nanowires may represent the ultimate size limit in exploring induced phase transitions in nanoscale systems that no other existing synthesis method will be able to afford.

To be able to realise the potential that these system have to offer, the fundamental behaviour in terms of the structure and electronic properties of these materials restricted to the smallest possible scale needs to be fully understood.

We report here that GeTe, a phase change material, currently considered to be one of the most promising materials for data-storage applications [10], can efficiently be encapsulated within SWNTs. Information on the structure of the encapsulated nanowires extracted from high resolution transmission electron microscopy, in addition to electronic structure information of the hybrid systems from photoemission experiments will be discussed in this paper.

\section{EXPERIMENT}

Arc-discharge SWNTs with a narrow diameter distribution centered around $1.4 \mathrm{~nm}$ were used in this study after air thermal annealing at $450{ }^{\circ} \mathrm{C}$, employed to remove amorphous carbon and $-\mathrm{COOH}$ groups present on the surface of nanotubes after acid purification.

The filling procedure employed in this study exploits the capillary properties of carbon nanotubes and it is based on the ability of the molten filling material to wet the surface of the nanotube [15]. SWNTs were heated in a quartz tube sealed under vacuum with the molten filling material to $100{ }^{\circ} \mathrm{C}$ above the melting point of GeTe and allowed to cool down slowly to room temperature. Transmission electron microscopy (TEM) and scanning TEM (STEM) were used to validate the presence of the filling material within SWNTs and assess the filling yield of the sample.

Ultraviolet photoelectron spectroscopy (UPS) measurements were carried out using He I radiation $(21.22 \mathrm{eV})$ from an Omicron HIS13 windowless He lamp, on an Omicron Multiprobe UHV system equipped with an EA125 hemispherical photoelectron energy analyser. All spectra were recorded at room temperature, with an overall resolution better than $190 \mathrm{meV}$, determined from the Fermi edge of a clean Au film. An electron pass energy of $5 \mathrm{eV}$ and an analysis area of $2 \mathrm{~mm}$ diameter determined by the entrance slit of the photoelectron analyzer has been used. For UPS measurements, films were prepared by drop casting the nanotube material directly onto the supporting sample plate, after sonication in acetone and subsequent ultracentrifugation in order to remove excess GeTe material left after the filling process.

\section{DISCUSSION}

The filled material was examined using the STEM and representative images are presented in figure 1. In the bright field images (figure 1a), carbon nanotubes appear with a 
contrast similar to that in conventional TEM images, whereas in the high-angle annular dark field images ( $Z$ contrast images - figure $1 \mathrm{~b}$ ), the filling species which have high atomic number $\mathrm{Z}$ compared to the encapsulating $\mathrm{C}$ are imaged as discrete, bright wires due to stronger incoherent scattering of electrons.

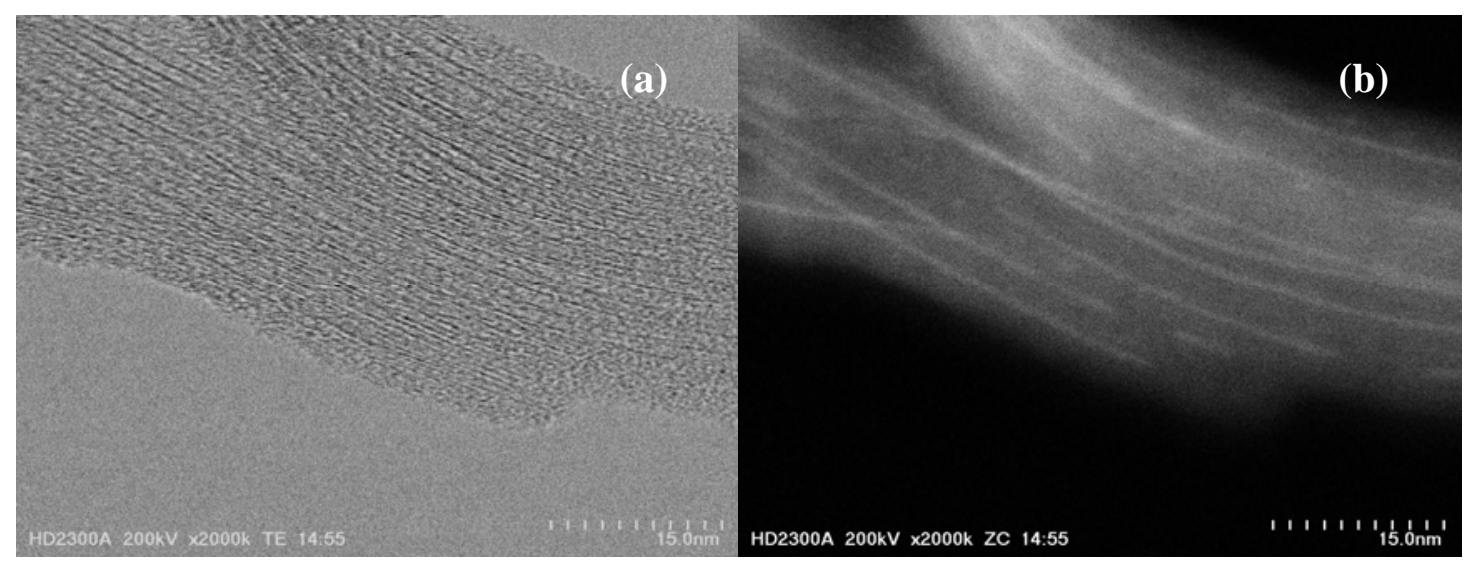

Figure 1. Bright field (a) and the corresponding high-angle annular dark field (b) STEM images showing GeTe encapsulated within single-walled carbon nanotubes.

High resolution TEM data indicating that GeTe have been effectively incorporated within SWNTs is presented in figure $2 \mathrm{a}$. Additionally, energy dispersive $\mathrm{X}$-ray spectroscopy (EDX) analysis indicates the presence of the filling elements Ge and Te within the sample (spectrum in figure $2 b$ ).
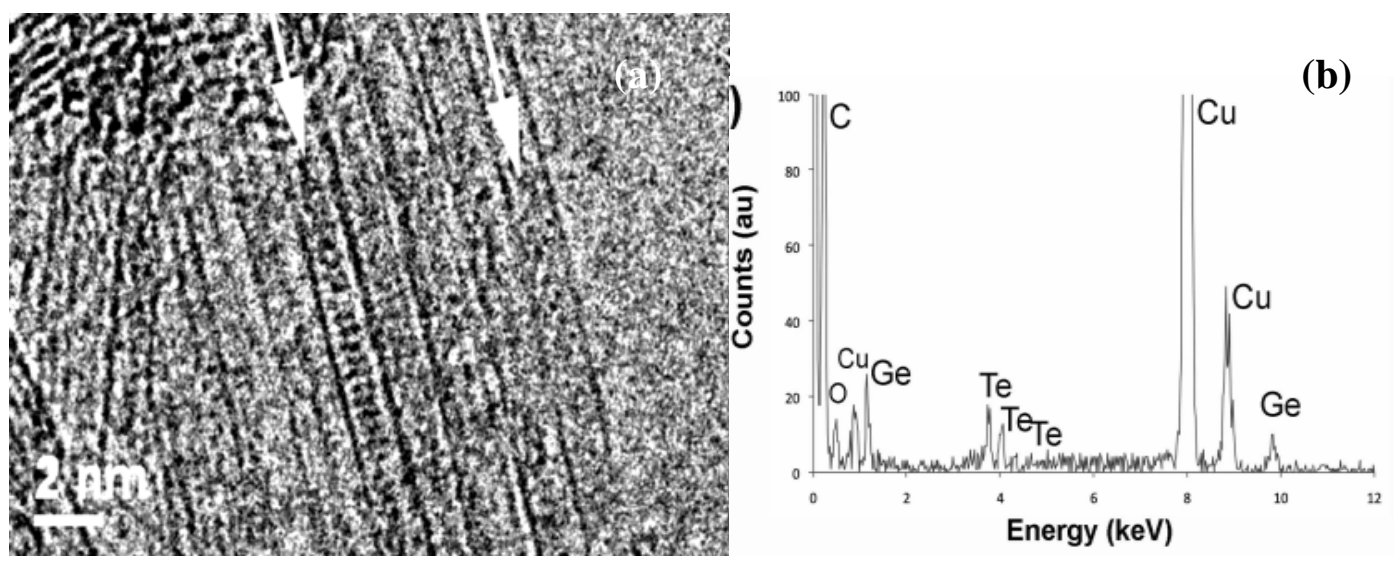

Figure 2. HRTEM image of a bundle of SWNTs containing amorphous (right arrow) and crystalline (left arrow) GeTe filling (a). (b) Energy dispersive X-ray spectroscopy (EDX) spectra obtained from the same region as the image in (a) confirming the identity of the filling.

Within the inner cores of nanotubes GeTe forms continuous nanowires with lengths up to a few hundred $\mathrm{nm}$. Based on a statistical analysis of the TEM data, a filling yield in excess of 80 
$\%$ has been estimated. Variable crystallisation is found, possibly correlated with the nanotubes diameter, as indicated in figure $1 \mathrm{a}$, where both amorphous and crystalline filling with a $0.3 \mathrm{~nm}$ lattice spacing is observed within the same sample.

Photoemission spectroscopy has been utilised to extract information on the electronic band structure of the filled nanotubes. The electronic structure is depicted in figure $3 \mathrm{a}$, where the valence band spectra of both the filled and pristine nanotubes, taken at excitation energy of 21.22 $\mathrm{eV}$ (He I) are shown. In order to enable a quantitative comparison, the spectra have been normalised to the secondary electron peak and the Shirley background subtracted.
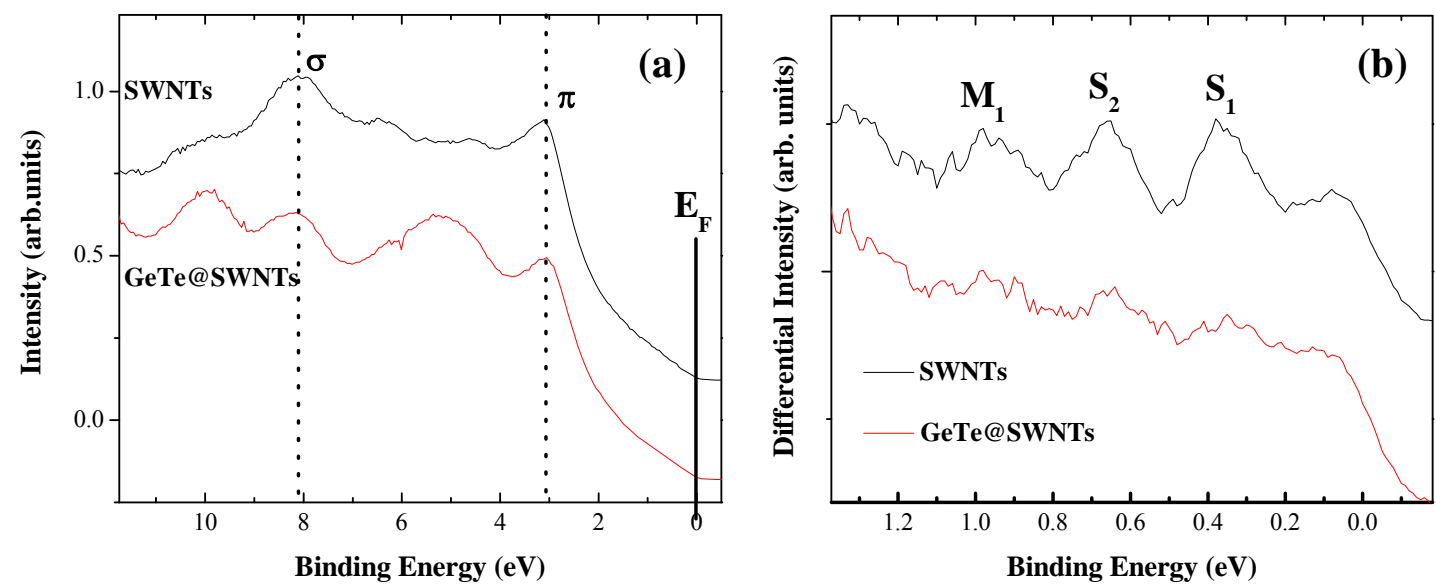

Figure 3 (a) UPS spectra of pristine and filled SWNTs. Energies are defined with respect to the Fermi energy $E_{F}$ and spectra are shifted vertically for clarity. Dotted vertical lines indicate the maximum position of the $\pi$ and $\sigma$ bands of SWNTs. (b) Valence band spectra near the Fermi energy for pristine and filled nanotubes, highlighting the presence of van Hove singularities $\left(\mathrm{S}_{1}\right.$, $\mathrm{S}_{2}, \mathrm{M}_{1}$ ) in the DOS, typical to one-dimensional systems.

The spectrum measured on the pristine nanotubes shows the well-known features characteristic to the three-fold coordination of $\mathrm{C}$ atoms: the $\sigma$-bands corresponding to the strong in-plane bonding situated at $\sim 8.1 \mathrm{eV}$ and the $\pi$-bands at $\sim 3.1 \mathrm{eV}$, representative of the weak bonding perpendicular to the graphene plane.

The valence band structure of GeTe-filled SWNTs displays some features that could be attributed to the filling material. Previous UPS measurements of bulk GeTe indicate a subband from 0 to $6 \mathrm{eV}$, in the $\mathrm{Ge} 4 \mathrm{p}$ region, that contains some fine structures at around $1.8 \mathrm{eV}, 3.5 \mathrm{eV}$ and $4.9 \mathrm{eV}$, respectively [16]. The $4.9 \mathrm{eV}$ feature of the subband is visible in our photoemission spectrum, however we do not observe the feature at $1.8 \mathrm{eV}$, possibly due to a different local structure of the GeTe confined within the nanotubes with respect to the bulk material, that could modify the electronic structure and give a different photoemission response. The feature at 3.5 $\mathrm{eV}$ is obscured by the presence of the $\pi$ states of the SWNTs, as is the lower Ge $4 \mathrm{~s}$ subband at around $8 \mathrm{eV}$, by the $\sigma$ states of the SWNTs existing in the same region.

No energy shift of the $\pi$ bands of the filled sample with respect to the pristine SWNTs is observed, suggesting the absence of charge transfer between the tubes and the filling. According to a rigid band shift model, electron transfer from the nanotubes to the filling material would lead to a shift of the Fermi level towards the valence band [17]. 
To assess the low energy electron properties of the filled nanotubes, a detailed analysis of the region in the close vicinity of the Fermi energy has been undertaken. Photoemission spectra recorded in the region with binding energies of up to $1.3 \mathrm{eV}$ from the Fermi level of both filled and pristine nanotubes reveals the distinct structures originating from the one-dimensional character of single-walled nanotubes, corresponding to the well-known van Hove singularities (vHs), depicted in the differential spectra in figure 3b. For pristine SWNTs, three peaks are distinctly resolved and correspond to the first two vHs of semiconducting nanotubes $\left(\mathrm{S}_{1}\right.$ and $\left.\mathrm{S}_{2}\right)$ and to the first vHs of metallic nanotubes $\left(\mathrm{M}_{1}\right)$, respectively. They are observed at binding energies of $0.37 \mathrm{eV}\left(\mathrm{S}_{1}\right), 0.66 \mathrm{eV}\left(\mathrm{S}_{2}\right)$ and $0.96 \mathrm{eV}\left(\mathrm{M}_{1}\right)$, respectively, with a full width at half maximum of about $0.1 \mathrm{eV}$. Upon filling, these peaks broaden and appear smeared out compared to the ones of pristine nanotubes, however they do not display a visible shift in binding energy, consistent with the absence of charge transfer between the nanotube and the filling as indicated above, suggesting furthermore that the electronic properties of SWNTs and the filling are not perturbed by each other.

We however record changes in the work function of the filled nanotubes with respect to the pristine ones. We determine the work function using the secondary electron cut-off of the electron spectra, since the electrons responsible for the secondary edge have just sufficient energy to overcome the workfunction of the material. The work function determined by the intersection of the high binding energy cut-off of the accelerated electrons with the base line of the spectra obtained for pristine SWNTs is $4.8 \mathrm{eV}$, consistent with previous studies [18]. Upon filling, a reduction in the work function by $0.4 \mathrm{eV}$ is observed in the case of GeTe-filled nanotubes. The reduction can be attributed to the presence of an electric dipole at the interface between the nanotube and the filling, which changes the energy an electron needs to leave the sample.

\section{CONCLUSIONS}

We report on the encapsulation of GeTe within the inner cores of SWNTs and show that continuous GeTe nanowires of uniform morphology are formed, in contrast to catalyst-assisted vapour-liquid-solid synthesis methods which result in various mixed morphologies (straight, helical, etc. nanowires). The catalyst-grown structures often contain other impurities and always exhibit an oxide layer on the surface of the nanowire, whereas with our method the protective shell of the nanotube prevents the oxidation of the encased nanowire and maintains its chemical composition due to the inertness of the surrounding nanotube.

Photoemission spectroscopy shows no charge transfer between the encased nanowires and the nanotubes, suggesting that their electronic properties are not affected by each other's presence. Owing to the reduced dimensionality of the encapsulated nanostructure a lower melting point is expected compared to that of the bulk material $\left(725^{\circ} \mathrm{C}\right)$, therefore advantageous for the reduction in the programming energy required for phase switching.

Due to the small integrated structure, GeTe-filled nanotubes present potential for dense memory arrays and open up interesting possibilities for more compact storage devices where the encasing nanotubes act as the interconnects that provides the means for effectively controlling the encapsulated nanowires so as to store data and to access the stored data and impede heat or electricity transfer between the phase change nanowires. This will prevent adjacent intercell 
thermal interferences that can corrupt data due to leakage current and excessive heat, issues well known to preclude future scaling of dense memory arrays.

Future work will explore aspects related to the smallest nanotube diameter for which reversible phase changes of the encased nanowire can be accomplished, together with a pronounced contrast in electrical resistivity between the amorphous and the crystalline state and threshold switching behaviour, most useful for future non-volatile memory applications.

\section{REFERENCES}

1. E. Bichoutskaia, A. M. Popov, Y. E. Lozovik, Materials Today, 11, 6, 38, (2008).

2. T. Rueckes, et al. Science 289, 94, (2000).

3. R. Carter, J. Sloan, A.I. Kirkland, et al, Phys. Rev. Lett. 96, 215501, (2006).

4. R.R. Meyer et al., Science 289, 1324, (2000).

5. E. Philp, J. Sloan, A.I. Kirkland, R.R. Meyer, S. Friedrichs, J.L. Hutchison, M.L.H. Green, Nature Mater., 2, 788 (2003).

6. J. Sloan, R. Carter, et al., Microscopy of Semic. Mat. 120, 213, (2008).

7. M. Chen, K. A. Rubin, R. W. Barton, Appl. Phys. Lett. 49, 9, 502, (1986).

8. M. H. Lankhorst, B. W. Ketelaars, and R. A. Wolters, Nature Mater. 4, 266, (2005).

9. H. B. Chung, K. Shin, J. M. Lee, J. Vac. Sci. Technol. A 25, 1, 48, (2007).

10. M. Wuttig, N. Yamada, Nature Mater. 6, 824, (2007).

11. S. Raoux et al., IBM J. Res. \& Dev. 52, 4/5, July/September, (2008).

12. S. Raoux, Annu. Rev. Mater. Res. 39, 25, (2009).

13. S.-H. Lee, Y. Jung, R. Agarwal, Nature Nanotech. 2, 626, (2007).

14. S. Raoux, C. T. Rettner, J. L. Jordan-Sweet, A. J. Kellock,T. Topuria, P. M. Rice, and D. C. Miller, J. Appl. Phys. 102, 9, 94305 (2007).

15. J. Sloan, A. Kirkland, J. L. Hutchison, and M. L. H. Green, Chem. Commun. 1319 (2002).

16. S. Hosokawa, Y. Hari, T. Kouchi, I. Ono, H. Sato, M. Taniguchi, A. Hiraya, Y. Takata, N. Kosugi, M. Watanabe, J. Phys.: Condens. Matter 10, 1931, (1998).

17. H. Rauf, H. Shiozawa, T. Pichler, M. Knupfer, B, Buchner, H. Kataura, Phys. Rev. B 72, $245411(2005)$.

18. S. Suzuki, C. Bower, Y. Watanabe, O. Zhou, Appl. Phys.Lett. 76 4007, (2000). 